\title{
SMAC-Survivin apoptotic "switch" is regulated through the PI3K-Src-p at the a1-Na/K-ATPase in NASH related hepatocellular carcinoma: Studies in-vitro/vivo and in humans
}

\author{
Juan SANABRIA*
}

Department of Surgery, Marshall and Case Western Reserve University, Huntington and Cleveland, OH, USA

Introduction: Hepatocellular carcinoma (HCC) is the second and fastest-growing cause of cancer related mortality worldwide. Our group has described the signaling properties of the $\alpha 1-\mathrm{Na} / \mathrm{K}-\mathrm{ATP}$ ase providing a pathway for organogenesis during cell development. We hypothesized that during hepatocarcinogenesis, regulation of Src-phosphorylation at the al-subunit of the NKA causes an imbalance of the Smac/DIABLO-Survivin apoptotic signaling process, favoring cell division and tumorigenesis.

Methods: Expression of Cav-1/Smac/Survivin proteins was performed on immuno-stained HCC cell lines (Hep3 \& SNU475), and livers from a NASH-HCC rodent model, and humans. Furthermore, signaling pathway studies were explored in-vitro guided by RNA sequencing. Selective blockage of Src-phosphorylation at its kinase domain was performed by administration of a synthetic peptide $(33 \mathrm{aa}=$ pNaKtide, developed from $\mathrm{N}$ domain of Na/K-ATPase). Significant differences among groups were accepted at $p<0.05$ using ANOVA/t-test.

Results: $\alpha 1-N K A / S r c-p$ inhibition promoted apoptosis of cell lines, and receptor' IC50 drove concomitantly both, Survivin's downregulation and SMAC's upregulation expressions (dose-related, $p<0.01$ ). In-vivo, liver tumor burden was significantly lower in animals treated with pNaKtide $(p<0.01)$, and the expressions of Cav-1/Survivin were significantly higher in liver tumor tissue from non-treated when compared to treated animals $(p<0.01)$. A similar pattern of Cav-1 and survivin expressions was noted in tumors from patients with NASH \pm HCC when compared to liver tissue from healthy subjects or subjects with liver metastases $(p<0.05)$. In-vitro, Src-p at the a1-NKA activates PI3K/Akt dependent and independent to EGFr/Grb2 pathways.

Conclusions: $\alpha 1-N K A / S r c-p$ in Caveola regulates Survivin/SMAC expressions which in turn modulate a cellular "switch" from apoptosis to cell division involving two signaling pathways. 